\title{
Adesão ao tratamento farmacológico e não farmacológico e fatores associados na atenção primária da hipertensão arterial
}

\author{
Adherence to pharmacological and non pharmacological treatment \\ for arterial hypertension and associated factors in primary care
}

Edmarlon Girotto ${ }^{1}$

Selma Maffei de Andrade ${ }^{2}$

Marcos Aparecido Sarria Cabrera ${ }^{3}$

Tiemi Matsuo ${ }^{4}$

\footnotetext{
Departamento de Ciências Farmacêuticas, Centro de Ciências da Saúde, Universidade Estadual de Londrina. R. Armando Tonelli 189, Jardim Vila Romana I. 86200-000 Ibiporã PR.

eddieuel@yahoo.com.br ${ }^{2}$ Departamento de Saúde Coletiva, Programa de PósGraduação em Saúde Coletiva, Centro de Ciências da Saúde, Universidade Estadual de Londrina.

${ }^{3}$ Departamento de Clínica Médica, Programa de PósGraduação em Saúde Coletiva, Centro de Ciências da Saúde, Universidade Estadual de Londrina.

${ }^{4}$ Departamento de Estatística, Programa de Pós-Graduação em Saúde Coletiva e em Ciências da Saúde, Centro de Ciências da Saúde, Universidade Estadual de Londrina.
}

\begin{abstract}
A cross-sectional study was conducted with 385 hypertensive patients between 20 and 79 years of age in a family health unit in Londrina, State of Parana, Brazil, to determine the adherence to pharmacological and non pharmacological treatment for arterial hypertension in primary healthcare and identify associated factors. The adherence to pharmacological treatment was 59\%. Regular physical activity was reported by $68(17.7 \%)$ hypertensive patients and 266 (69.1\%) mentioned dietary changes. In the multiple regression analysis, adherence to drug treatment was associated with: increased age (50 to 79), consulting with a physician at least once a year, no regular alcohol consumption and previous myocardial infarction. Hypertensive males, with education up to $4^{\text {th }}$ grade and above, who had at least one consultation per year, with diabetes or no history of high cholesterol, were more adherent to physical activity. Dietary changes were associated with education below the $4^{\text {th }}$ grade and consulting with a physician at least once a year. These results show variable levels of adherence to treatment and demonstrate the importance of comprehensive actions in the care for hypertensive patients.
\end{abstract}

Key words Adherence to pharmacological treatment, Hypertension, Primary healthcare, Risk factors
Resumo Com o objetivo de determinar a adesão aos tratamentos farmacológico e não farmacológico da hipertensão arterial na atenção primária e identificar fatores associados, realizou-se estudo transversal com 385 hipertensos de 20 a 79 anos cadastrados em uma unidade de saúde da família de Londrina, Paraná. A adesão ao tratamento farmacológico foi de 59,0\%. A atividade física regular foi relatada por $68(17,7 \%)$ hipertensos, enquanto $266(69,1 \%)$ referiram mudanças da alimentação. $\mathrm{Na}$ análise de regressão múltipla, associaram-se à adesão ao tratamento farmacológico: maior idade (50 a 79 anos), ter recebido ao menos uma consulta ao ano, não ingestão regular de bebidas alcoólicas e infarto prévio. Hipertensos do sexo masculino, com escolaridade igual ou superior à $4^{a}$ série, que receberam pelo menos uma consulta ao ano, com diabetes e sem relato de colesterol elevado mostraram-se mais aderentes à atividade física regular. A realização de dieta associou-se à escolaridade inferior à $4^{a}$ série e ao fato de ter recebido no mínimo uma consulta ao ano. Tais resultados demonstram níveis variados de adesão ao tratamento e evidenciam a importância de ações integrais na atenção ao hipertenso.

Palavras-chave Adesão ao tratamento farmacológico, Hipertensão, Atenção Primária à Saúde, Fatores de Risco 


\section{Introdução}

As doenças do aparelho circulatório representam a principal causa de morbimortalidade na sociedade contemporânea, destacando-se a doença coronariana, as cerebrovasculares e a insuficiência cardíaca ${ }^{1,2}$. A hipertensão arterial (HA) é o principal fator de risco para essas doenças ${ }^{3}$.

São muitos os fatores identificados que contribuem para a elevação dos níveis pressóricos, entre os quais se destacam idade avançada, etnia negra, obesidade, consumo excessivo de álcool, sedentarismo, dislipidemias, diabetes mellitus e alto teor de sódio na alimentaçãa ${ }^{1,4,5}$. Deste modo, para tornar o controle da hipertensão mais eficaz, torna-se indispensável, além do seu tratamento, também o controle de seus fatores de risco.

Um dos maiores desafios no combate à hipertensão arterial ainda se deve à não adesão ao tratamento ${ }^{6}$. Estudos mostram baixos níveis de adesão à terapia anti-hipertensiva, além de os maiores índices estarem associados a serviços de saúde especializados ${ }^{7}$. A adesão, segundo Leite e Vasconcelos ${ }^{6}$, corresponde à concordância entre a prescrição médica e a conduta do próprio paciente. Porém, são muitos os fatores que contribuem para a falta de adesão, tais como as dificuldades financeiras, o maior número de medicamentos prescritos, o esquema terapêutico, os efeitos adversos dos medicamentos, a dificuldade de acesso ao sistema de saúde, a inadequação da relação médico-paciente, a característica assintomática da doença e a sua cronicidade ${ }^{6,8}$.

Outras formas de manejo da hipertensão arterial, como a prática de atividade física e a dieta também são importantes'. Alguns estudos mostram baixa adesão a essas práticas na população em geral, e em grupos específicos, como os hipertensos e diabéticos, mais sujeitos aos efeitos danosos do sedentarismo e da dieta não adequada. Em pesquisa realizada com hipertensos e/ou diabéticos de Francisco Morato (SP), foram identificados apenas $33,3 \%$ e $42,2 \%$ de indivíduos com dieta adequada e parcialmente adequada, respectivamente, e somente $25,0 \%$ realizavam atividade física de forma regular ${ }^{10}$. Investigação com hipertensos cadastrados no programa Hiperdia verificou que a restrição de consumo de sal é o principal artifício alimentar utilizado para o controle da hipertensão $(63,0 \%)$, seguido da redução do consumo de gorduras $(21,0 \%)$ e açúcar e doces $(8,0 \%)^{11}$.

As mudanças de comportamentos necessárias para o controle da pressão arterial são desafiadoras para hipertensos e serviços de saúde. Es- tudos que identifiquem as prevalências de adesão às diferentes modalidades de tratamento $\mathrm{e}$ os grupos populacionais mais vulneráveis à não adesão são importantes para direcionar ações individuais e coletivas de atenção à saúde. Neste contexto, este estudo buscou determinar a adesão ao tratamento anti-hipertensivo farmacológico e não farmacológico na atenção primária e identificar fatores associados em população hipertensa do município de Londrina, Paraná.

\section{Metodologia}

Este estudo transversal, exploratório, foi realizado na área de abrangência de uma Unidade de Saúde da Família de Londrina, Norte do Paraná. Participaram do estudo hipertensos com diagnóstico há, no mínimo, seis meses, e com idade entre 20 e 79 anos.

Para definição da população de estudo, foram identificados os hipertensos no Sistema de Cadastramento e Acompanhamento de Hipertensos e Diabéticos (Hiperdia), no Sistema de Informação da Atenção Básica (Siab) e em fichas de aprazamento, as quais são usadas, nesse serviço, para acompanhamento e agendamento de retornos dos hipertensos. A partir do cruzamento dessas três fontes de informações, obteve-se um número de 695 hipertensos. Considerando um erro de 3,5\%, nível de confiança de $95 \%$ e prevalência de $50 \%$, determinou-se uma amostra de 442 indivíduos (incluída estimativa de perdas ou exclusões de $20 \%$ ).

A amostragem foi sistemática e aleatória, na listagem final de hipertensos ordenada por sexo e idade, para garantir proporcionalidade. Do total de amostrados, 52 foram excluídos, por mudança de endereço para área de outra USF (33), óbito (6), estar fora da faixa etária definida (1) e não ter histórico de hipertensão (12). Para a análise do tratamento farmacológico também foram excluídos hipertensos aos quais não foram prescritos medicamentos para o controle da pressão arterial (29). Os dados foram coletados por meio de entrevistas nos domicílios dos selecionados, de janeiro a junho de 2007, em até cinco visitas, realizadas por entrevistadores treinados e avaliados. Previamente à coleta de dados, foi realizado estudo piloto, com população diversa à selecionada, porém com características semelhantes, para verificar a adequação do formulário de entrevista construído especificamente para esta pesquisa, que foi baseada em revisão da literatura sobre o tema. Foram reentrevistadas 10\% 
da amostra, com vistas a garantir a confiabilidade dos dados, sendo evidenciada alta concordância das informações.

As variáveis relativas ao tratamento não farmacológico foram: atividade física regular realização de exercícios dinâmicos (caminhada, ciclismo, dança, ginástica, entre outras) com frequência mínima de três vezes na semana e duração mínima de 30 minutos cada sessão, conforme recomendações da VI Diretrizes Brasileiras de Hipertensão Arterial $^{1}$ e dieta - qualquer mudança nos hábitos alimentares para ajudar no controle da hipertensão arterial. Com relação a estes, foram identificados os principais alimentos de risco que apresentaram redução em seu consumo.

A adesão ao tratamento farmacológico foi categorizada da seguinte forma: hipertensos que abandonaram todo o esquema terapêutico prescrito foram enquadrados como não aderentes, aqueles que tomavam os medicamentos, porém de forma irregular ou que deixavam de tomar um dos medicamentos prescritos, foram classificados como parcialmente aderentes, e os que referiram utilizá-los de forma correta, como totalmente aderentes. $\mathrm{Na}$ análise dicotômica, o grupo dos totalmente aderentes (adesão = sim) foi comparado aos grupos não aderentes e parcialmente aderentes (adesão = não). Também foram identificados os principais motivos alegados pelos entrevistados para a não adesão, total ou parcial.

As variáveis independentes foram: sexo (masculino e feminino); faixa etária ( 20 a 49 anos e 50 a 79 anos); escolaridade (até $3^{\text {a }}$ série e $4^{\text {o }}$ série ou mais); trabalho remunerado ( $\operatorname{sim}$ ou não); classificação econômica, baseada na proposta da Associação Brasileira de Empresas de Pesquisa (ABEP) por meio do Critério de Classificação Econômica Brasil ${ }^{12}$, com classificação em classes A, B ou C (melhores condições econômicas) e D ou E (piores condições econômicas); acesso a plano de saúde (sim ou não); opinião sobre o atendimento da USF (ótimo/bom ou regular/ ruim/péssimo); verificação da PA (mínimo de uma medida ao mês ou nunca/menos de uma medida ao mês); consultas médicas (mínimo de uma consulta ao ano ou nunca/menos de uma consulta ao ano); tabagismo - tabagismo atual ( sim ou nunca fumou/ex-tabagista), sendo considerados ex-tabagistas os que abandonaram o hábito há 12 meses ou mais; ingestão regular de bebidas alcoólicas, considerado como ingestão em pelo menos três dias na semana, independente da quantidade consumida (sim ou não); comorbidades (autorreferidas) - foram consi- deradas, se respondidas afirmativamente pelo entrevistado: diabetes, colesterol elevado e doenças cardiovasculares (antecedentes de infarto e/ ou acidente vascular cerebral). Além dessas, a atividade física regular e a dieta também foram consideradas variáveis independentes para o tratamento farmacológico.

Este estudo foi aprovado pelo Comitê de Ética em Pesquisa da Universidade Estadual de Londrina. Os entrevistados foram informados quanto aos objetivos da pesquisa e, após leitura, entendimento e assinatura do termo de consentimento livre e esclarecido, responderam às questões do instrumento de coleta de dados.

Todos os formulários de coleta foram codificados, duplamente digitados e comparados para a correção dos erros de digitação pelo programa Epi Data 3.1 para Windows. A tabulação dos dados foi realizada com o uso do programa Epi Info, versão 3.3.2, com análises descritivas (números absolutos, percentuais, medidas de tendência central e dispersão). O teste Qui-quadrado ou exato de Fisher foi usado nas análises bivariadas de variáveis categóricas. No modelo de regressão com múltiplas variáveis, realizado no programa SPSS (Statistical Package for Social Science) versão 15.0 (SPPS Inc., Chicago, USA), todas as que apresentaram valor de $\mathrm{p}<0,20$ na análise bivariada foram testadas e, no modelo final após a seleção de variáveis pelo método stepwise, permaneceram apenas aquelas estatisticamente significativas $(\mathrm{p}<0,05)$. Optou-se pelo modelo de regressão de Poisson multivariável com variância robusta para a estimação da razão de prevalências (RP) devido à elevada prevalência dos desfechos adesão ao tratamento farmacológico e dieta.

\section{Resultados}

Dos 390 hipertensos elegíveis para o estudo, quatro não foram localizados e um recusou-se a participar da pesquisa. Ao final, foram estudadas 385 pessoas $(98,7 \%)$, com idade média de 58,9 anos $\pm 12,0$ (mínima de 22 e máxima de 79 anos), sendo 241 mulheres $(62,6 \%)$. Os homens apresentaram idade média de 59,0 anos $\pm 12,3$, e as mulheres, 58,8 anos $\pm 11,8$.

Aproximadamente metade dos hipertensos $(49,6 \%)$ referiu ter estudado até a $3^{\text {a }}$ série do ensino fundamental, $42,6 \%$ mencionaram trabalho remunerado e $45,9 \%$ foram enquadrados nas classes econômicas D ou E. Acesso a plano de saúde foi citado por 23,1\%. Quanto ao atendimento re- 
cebido pela USF, 66,8\% o consideraram ótimo ou bom. A verificação da pressão arterial em intervalos mínimos de um mês e o recebimento de pelo menos uma consulta médica ao ano foram referidos, respectivamente, por $77,4 \%$ e $56,6 \%$ dos hipertensos avaliados. O tabagismo atual e a ingestão regular de bebidas alcoólicas foram relatados por $16,9 \%$ e $5,5 \%$ dos entrevistados, respectivamente. Entre as comorbidades avaliadas, $28,6 \%$ mencionaram colesterol elevado, $22,9 \%$ diabetes, e $15,3 \%$ infarto ou AVC prévios.

A prática de atividade física foi relatada por $114(29,6 \%)$ hipertensos, mas apenas $68(17,7 \%)$ referiram praticá-la regularmente. Mudanças na alimentação (dieta) foram relatadas por 266 $(69,1 \%)$. Destes, a grande maioria (99,6\%) alegou redução do consumo de alimentos de risco, sendo a restrição do consumo de sal $(84,2 \%)$, gorduras $(36,2 \%)$ e doces $(26,0 \%)$ os mais referidos.

A adesão ao tratamento farmacológico foi analisada em 356 hipertensos, uma vez que 29 $(7,5 \%)$ foram excluídos por não terem recebido prescrição médica de medicamentos para o controle da pressão arterial. Destes, $32(9,0 \%)$ foram considerados não aderentes e $114(32,0 \%)$ parcialmente aderentes. Deste modo, 210 hipertensos $(59,0 \%)$ foram considerados aderentes ao tratamento farmacológico. Os principais motivos alegados para a não adesão foi o esquecimento $(32,2 \%)$, achar que a pressão arterial estava controlada (21,2\%), os efeitos adversos dos medicamentos $(13,7 \%)$ e não apresentar sintomas (11,0\%). Também foram relevantes os motivos indisponibilidade de medicamentos na unidade de saúde (7,5\%), não querer tomar medicamentos pelo resto da vida (7,5\%), utiliza-os apenas quando se sente mal (6,8\%), e ingestão de bebidas alcoólicas $(6,2 \%)$.

Nas análises bivariadas de associações com a adesão ao tratamento não farmacológico (Tabela 1), mostraram-se mais aderentes à realização de atividade física regular os hipertensos que referiram diabetes $(25,0 \%)$, do sexo masculino $(22,9 \%)$, com maior escolaridade $(22,2 \%)$, que recebiam atendimento médico pelo menos uma vez ao ano $(22,0 \%)$, que relataram colesterol não elevado $(20,4 \%)$ e que tinham a pressão arterial verificada pelo menos uma vez ao mês $(20,1 \%)$. A adesão à dieta foi mais frequente entre hipertensos com menor escolaridade (77,5\%), com acesso a plano de saúde $(77,5 \%)$, do sexo feminino $(73,9 \%)$, que recebiam atendimento médico pelo menos uma vez ao ano $(75,2 \%)$, que tinham a pressão arterial verificada pelo menos uma vez ao mês $(71,8 \%)$, que nunca fumaram ou eram ex-tabagistas $(71,9 \%)$ e que não consumiam bebidas alcoólicas regularmente (70,9\%) (Tabela 1).

$\mathrm{Na}$ análise bivariada da adesão ao tratamento farmacológico, verificou-se que hipertensos com acesso a plano de saúde $(71,1 \%)$, com trabalho não remunerado $(69,1 \%)$, com idade de 50 a 79 anos $(66,9 \%)$, que se consultavam com médico pelo menos uma vez ao ano $(67,3 \%)$ e que tinham sua pressão arterial medida pelo menos uma vez ao mês $(63,5 \%)$ são mais aderentes ao tratamento (Tabela 2). Também se mostraram mais aderentes ao tratamento farmacológico hipertensos que referiram infarto/ AVC prévios (78,7\%) e diabetes (71,6\%), que realizaram mudanças na alimentação para controle da pressão $(62,9 \%)$, que nunca fumaram ou eram ex-tabagistas $(62,2 \%)$ e que não consumiam bebidas alcoólicas regularmente $(61,1 \%)$ (Tabela 2$)$.

A Tabela 3 apresenta as variáveis que se conservaram associadas à adesão ao tratamento não farmacológico e farmacológico no modelo multivariado, controlando-se possíveis fatores de confusão. Observam-se maiores prevalências de atividade física regular entre hipertensos que não referiram colesterol elevado $(\mathrm{RP}=2,03)$, que se consultavam com médicos em intervalos máximos de um ano $(\mathrm{RP}=1,96)$, com maior escolaridade ( $4^{\mathrm{a}}$ série ou mais) $(\mathrm{RP}=1,78)$, que referiram diabetes $(\mathrm{RP}=1,74)$ e do sexo masculino $(\mathrm{RP}=1,52)$. No caso da dieta, esta associação se manteve apenas entre os hipertensos com menor escolaridade (até $3^{\mathrm{a}}$ série) $(\mathrm{RP}=1,26)$, e que se consultavam com médicos em intervalos máximos de um ano $(\mathrm{RP}=1,21)$. Mostraram-se mais aderentes ao tratamento farmacológico: hipertensos que não consumiam regularmente bebidas alcoólicas ( $\mathrm{RP}=2,62)$, com maior idade (50 a 79 anos) $(R P=2,37)$, que se consultavam com médicos em intervalos máximos de um ano (RP $=1,29)$ e que referiram infarto prévio $(\mathrm{RP}=1,25)$.

\section{Discussão}

Os resultados obtidos na presente investigação demonstraram níveis variados de adesão conforme as modalidades avaliadas. No que tange às medidas não farmacológicas, os baixos níveis de adesão à atividade física $(29,6 \%)$ tornam-se mais relevantes quando se observa que apenas $17,7 \%$ as realizam de forma regular, corroborando dados de outros estudos ${ }^{10,13}$. Segundo a Política Nacional de Promoção à Saúde, a oferta de práticas de atividade física deve fazer parte dos trabalhos efetuados pela rede básica de saú- 
Tabela 1. Associação entre adesão ao tratamento não farmacológico e variáveis independentes, em área de Unidade de Saúde da Família, Londrina, PR, 2007.

\begin{tabular}{|c|c|c|c|c|c|c|c|}
\hline \multirow{3}{*}{ Variáveis independentes } & \multirow{3}{*}{$\begin{array}{c}\text { Total } \\
(\mathbf{n}=385)\end{array}$} & \multicolumn{6}{|c|}{ Adesão Tratamento Não Farmacológico } \\
\hline & & \multicolumn{3}{|c|}{$\begin{array}{l}\text { Atividade Física Regular } \\
\quad(\mathrm{n}=68 ; 17,7 \%)\end{array}$} & \multicolumn{3}{|c|}{$\begin{array}{c}\text { Dieta } \\
(\mathrm{n}=266 ; 69,1 \%)\end{array}$} \\
\hline & & $\mathbf{n}$ & $\%$ & p & $\mathbf{n}$ & $\%$ & $\mathbf{p}$ \\
\hline \multicolumn{8}{|l|}{ Sexo } \\
\hline Masculino & 144 & 33 & 22,9 & $<0,050$ & 88 & 61,1 & $<0,050$ \\
\hline Feminino & 241 & 35 & 14,5 & & 178 & 73,9 & \\
\hline \multicolumn{8}{|l|}{ Faixa etária } \\
\hline 20 a 49 anos & 81 & 13 & 16,0 & 0,669 & 55 & 67,9 & 0,794 \\
\hline 50 a 79 anos & 304 & 55 & 18,1 & & 211 & 69,4 & \\
\hline \multicolumn{8}{|l|}{ Escolaridade } \\
\hline Até $3^{\mathrm{a}}$ série & 191 & 25 & 13,1 & $<0,050$ & 148 & 77,5 & $<0,001$ \\
\hline $4^{\mathrm{a}}$ série ou mais & 194 & 43 & 22,2 & & 118 & 60,8 & \\
\hline \multicolumn{8}{|l|}{ Trabalho remunerado } \\
\hline Sim & 164 & 22 & 13,4 & 0,060 & 56 & 34,1 & 0,236 \\
\hline Não & 221 & 46 & 20,8 & & 63 & 28,5 & \\
\hline \multicolumn{8}{|l|}{ Classificação econômica } \\
\hline A, B ou C & 208 & 35 & 16,8 & 0,641 & 146 & 70,2 & 0,612 \\
\hline D ou E & 177 & 33 & 18,6 & & 120 & 67,8 & \\
\hline \multicolumn{8}{|l|}{ Acesso a plano de saúde } \\
\hline Sim & 89 & 15 & 16,9 & 0,820 & 69 & 77,5 & $<0,050$ \\
\hline Não & 296 & 53 & 17,9 & & 197 & 66,6 & \\
\hline \multicolumn{8}{|l|}{ Atendimento da USF } \\
\hline Ótimo/bom & 257 & 50 & 19,5 & 0,191 & 172 & 66,9 & 0,193 \\
\hline Regular/ruim/péssimo & 128 & 18 & 14,1 & & 94 & 73,4 & \\
\hline \multicolumn{8}{|l|}{ Verificação da PA } \\
\hline Mínimo uma medida/mês & 298 & 60 & 20,1 & $<0,050$ & 214 & 71,8 & $<0,050$ \\
\hline Nunca ou menos uma/mês & 87 & 8 & 9,2 & & 52 & 59,8 & \\
\hline \multicolumn{8}{|l|}{ Consultas médicas } \\
\hline Mínimo uma consulta/ano & 218 & 48 & 22,0 & $<0,050$ & 164 & 75,2 & $<0,050$ \\
\hline Nunca ou menos uma/ano & 167 & 20 & 12,0 & & 102 & 61,1 & \\
\hline \multicolumn{8}{|l|}{ Tabagismo } \\
\hline Tabagismo atual & 65 & 12 & 18,5 & 0,853 & 36 & 55,4 & $<0,050$ \\
\hline Nunca ou ex-tabagista & 320 & 56 & 17,5 & & 230 & 71,9 & \\
\hline \multicolumn{8}{|c|}{ Ingestão regular de bebidas alcoólicas } \\
\hline Sim & 21 & 4 & 19,0 & 0,864 & 8 & 38,1 & $<0,050$ \\
\hline Não & 364 & 64 & 17,6 & & 258 & 70,9 & \\
\hline \multicolumn{8}{|l|}{ Diabetes } \\
\hline Sim & 88 & 22 & 25,0 & $<0,050$ & 63 & 71,6 & 0,563 \\
\hline Não & 297 & 46 & 15,5 & & 203 & 68,4 & \\
\hline \multicolumn{8}{|l|}{ Colesterol elevado } \\
\hline Sim & 110 & 12 & 10,9 & $<0,050$ & 81 & 73,6 & 0,222 \\
\hline Não & 275 & 56 & 20,4 & & 185 & 67,3 & \\
\hline \multicolumn{8}{|l|}{ Infarto/AVC prévios } \\
\hline Sim & 59 & 12 & 20,3 & 0,558 & 44 & 74,6 & 0,322 \\
\hline Não & 326 & 56 & 17,2 & & 222 & 68,1 & \\
\hline
\end{tabular}

$\mathrm{de}^{14}$, e são importantes medidas que busquem a intersetorialidade para a melhoria dos espaços públicos, visando ao estímulo, da população e de grupos mais vulneráveis, às práticas de atividade física. 
Tabela 2. Associação entre adesão ao tratamento farmacológico e variáveis independentes, em área de Unidade de Saúde da Família, Londrina, PR, 2007.

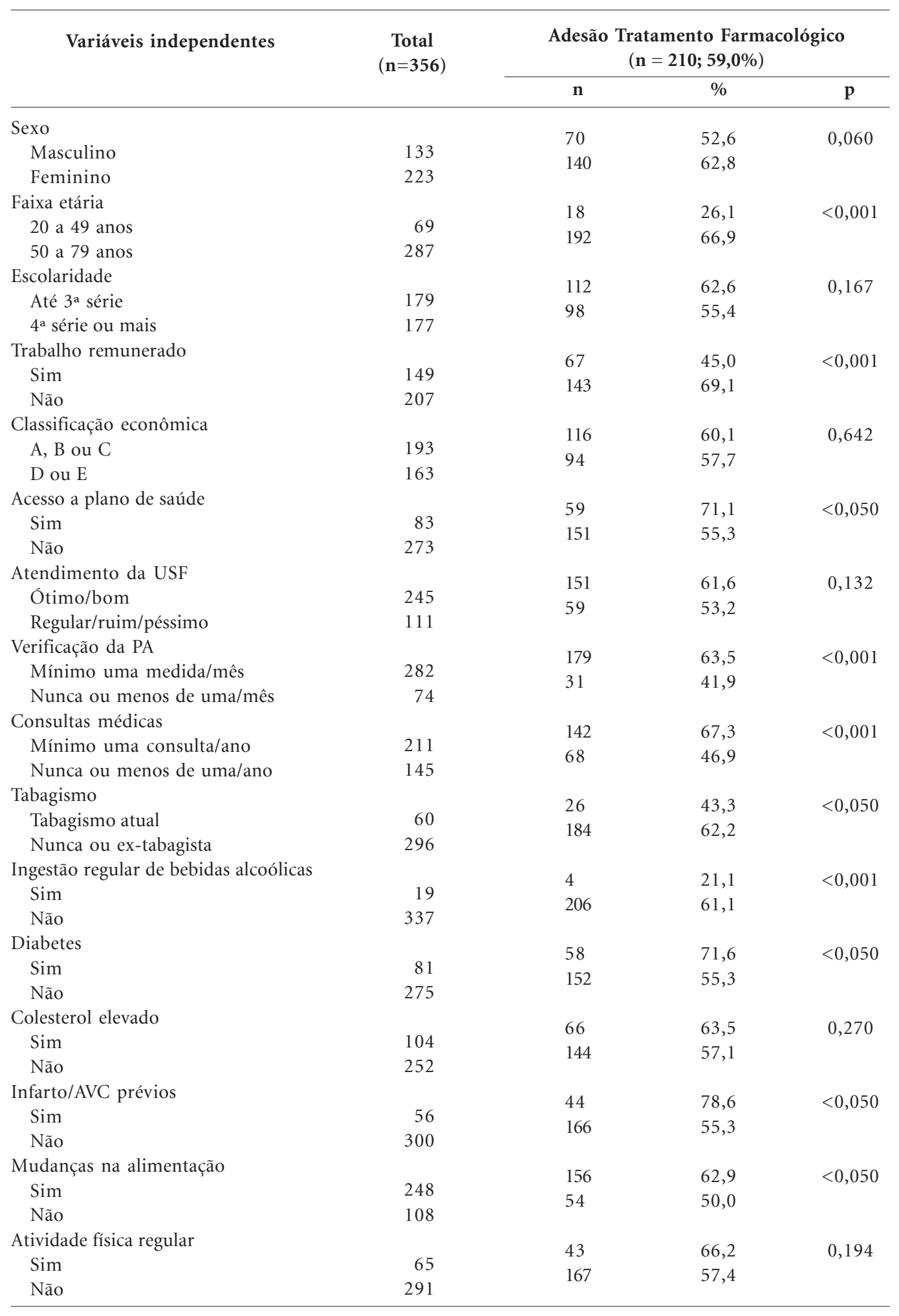


Tabela 3. Fatores associados à adesão ao tratamento farmacológico e não farmacológico segundo modelo de regressão de múltiplos fatores em área de Unidade de Saúde da Família, Londrina, PR, 2007*.

\begin{tabular}{|c|c|c|}
\hline \multirow{2}{*}{ Variáveis independentes } & \multicolumn{2}{|c|}{ Adesão ao Tratamento } \\
\hline & RP (IC95\%) & Valor $\mathrm{p}$ \\
\hline \multicolumn{3}{|l|}{ Atividade Física ${ }^{* *}$} \\
\hline Sexo (Masculino) & $1,52(1,01-2,29)$ & 0,048 \\
\hline Escolaridade (4a série ou mais) & $1,78(1,14-2,79)$ & 0,017 \\
\hline Consultas médicas (mínimo uma consulta/ano) & $1,96(1,23-3,11)$ & 0,004 \\
\hline Diabetes $(\operatorname{Sim})$ & $1,74(1,10-2,74)$ & 0,018 \\
\hline Colesterol elevado (Não) & $2,03(1,13-3,64)$ & 0,017 \\
\hline \multicolumn{3}{|l|}{ Dieta $^{* * *}$} \\
\hline Escolaridade (Até 3a série) & $1,26(1,10-1,44)$ & 0,001 \\
\hline \multicolumn{3}{|l|}{ Farmacológico $^{* * * *}$} \\
\hline Faixa etária (50 a 79 anos) & $2,37(1,58-3,54)$ & $<0,001$ \\
\hline Consultas médicas (mínimo uma consulta/ano) & $1,29(1,07-1,55)$ & 0,007 \\
\hline Ingestão regular de bebidas alcoólicas (Não) & $2,62(1,15-5,95)$ & 0,022 \\
\hline Infarto prévio (Sim) & $1,25(1,07-1,46)$ & 0,005 \\
\hline
\end{tabular}

redução do consumo dos alimentos de risco, como o de sal, assim como observado em outro estudo com hipertensos ${ }^{11}$. Deste modo, presume-se que os indivíduos associam o controle da hipertensão à diminuição desses produtos e não ao consumo de alimentos protetores, como frutas, verduras e legumes. Sturmer et al. ${ }^{15}$ verificaram que as recomendações médicas mais frequentes sobre mudanças alimentares são a redução da ingestão de sal e gorduras, sugerindo que medidas não farmacológicas ainda não estão totalmente incorporadas nas práticas educativas dos serviços e profissionais de saúde.

No presente estudo, a não adesão ao tratamento farmacológico, total ou parcial, foi de $41 \%$. Há grande variabilidade nos níveis de adesão ao tratamento farmacológico nos diferentes estudos, atribuída à falta de homogeneidade das metodologias utilizadas, que incluem distintas áreas, populações de hipertensos, tempos de seguimento, números de participantes e formas de obter ou sistematizar a informação sobre adesão ${ }^{16}$. Em estudo de validação de um questionário desenvolvido para medir não adesão de forma multidimensional, Santa Helena et al. ${ }^{17}$ observaram taxas de não adesão variando de $8,7 \%$ a $69,6 \%$, dependendo do método utilizado, sendo essa taxa mais alta quando usada uma medida combinada de contagem manual de medicamentos, perguntas de um questionário e valores de pressão arterial iguais ou superiores a 140 x $90 \mathrm{mmHg}$. Nesse mesmo estudo ${ }^{17}$, as taxas de não adesão medidas pelo emprego do questionário de Morisky-Green $^{18}(43,4 \%)$ e pela contagem manual de comprimidos $(43,4 \%)$ foram as que mais se aproximaram à observada na presente investigação.

Os principais motivos alegados por aqueles que não aderiam ao tratamento medicamentoso, esquecimento e achar que a pressão arterial estava controlada, revelam a necessidade de se estabelecer medidas que permitam ao hipertenso compreender adequadamente a hipertensão arterial e a importância da adaptação a uma situação que exige mudanças comportamentais contínuas e que favoreça o cumprimento das medidas terapêuticas indicadas ${ }^{19}$.

Em relação aos fatores associados à adesão encontrados nesta investigação, após controle de variáveis de confusão, a prática regular de atividade física mostrou-se mais prevalente no sexo masculino, concordando com resultados de outros estudos ${ }^{20,21}$. Salles-Costa et al. ${ }^{21}$ salientam que a alta jornada de trabalho semanal das mulheres, que inclui atividades domésticas, pode justificar esses achados. Vale lembrar que a atividade física, além de colaborar para o controle da hipertensão, também contribui para a melhora das condições físicas e psicológicas ${ }^{22}$, o que extrapola seus benefícios para além da prevenção de agravos cardiovasculares.

A escolaridade mostrou-se associada apenas à adesão ao tratamento não farmacológico, e a maior escolaridade mostrou associação positiva com a atividade física, concordando com outras 
investigações ${ }^{20-22}$. No entanto, a adesão à dieta associou-se à menor escolaridade. Pitanga e Lessa ${ }^{20}$ salientam que pessoas com menos escolaridade têm menos oportunidades de desenvolver atividades físicas no lazer. Quanto à dieta, vale destacar que a principal medida relatada pelos entrevistados foi a diminuição do consumo de sal. Talvez indivíduos com baixa escolaridade considerem a restrição do sal a principal medida alimentar para o controle da hipertensão. Todavia, a dificuldade de mensuração da adesão à dieta ${ }^{23}$, pela sua complexidade e dificuldade de dimensionar as reais alterações na alimentação, pode ter contribuído para um viés no que se refere à associação encontrada.

Neste estudo, observou-se maior adesão à atividade física regular entre hipertensos que referiram não ter colesterol elevado e, embora o delineamento transversal não permita inferir relação de causa e efeito, é possível que este tipo de adesão influencie nos níveis sanguíneos de colesterol. Guedes e Gonçalves ${ }^{24}$ encontraram menores níveis de colesterol total e LDL em pacientes com prática habitual de atividade física mais ativa. Além disso, estudo com jovens diabéticos participantes de acompanhamento educativo em São Paulo identificou que um programa de exercícios físicos melhora o perfil lipídico em curto prazo ${ }^{25}$.

Assim como em outras pesquisas ${ }^{26,27}$, este estudo identificou a maior faixa etária como condição associada à melhor adesão ao tratamento farmacológico. $\mathrm{O}$ aumento da idade está ligado à maior prevalência de doenças crônico-degenerativas, e isto pode tornar o indivíduo mais preocupado com seu estado de saúde, aumentando a adesão ao tratamento, uma vez que este processo depende, em grande parte, de como o hipertenso percebe sua condição de saúde. Essa preocupação também poderia justificar o fato de hipertensos que referiram infarto prévio e diabetes serem mais aderentes ao tratamento farmacológico e à prática regular de atividade física. A associação da adesão ao tratamento com doenças prévias, inclusive com a história familiar de doenças cardiovasculares, foi também verificada por Yahya et al. ${ }^{28}$, no Kuwait. É importante destacar que indivíduos que apresentam outras comorbidades, além da hipertensão, podem receber maior atenção dos profissionais e serviços de saúde, o que pode resultar em melhora nos índices de adesão ao tratamento farmacológico, bem como na prática de atividade física.

Detectou-se também, no presente trabalho, que a adesão ao tratamento farmacológico associou-se ao não consumo regular de bebidas alcoólicas. Isso denota que comportamentos de pro- teção à saúde estão fortemente associados a medidas de controle da hipertensão arterial, e que a redução do consumo de bebidas alcoólicas e combate ao tabagismo devem fazer parte do manejo da hipertensão arterial $^{1,29}$. O Sistema de Vigilância de Fatores de Risco e Proteção para Doenças Crônicas por Inquérito Telefônico (Vigitel) identificou que o consumo abusivo de álcool se correlacionou com outros comportamentos deletérios, como tabagismo, atividade física irregular e consumo de carnes com excesso de gordura ${ }^{30}$.

Neste trabalho, a maior frequência a consultas médicas associou-se às três modalidades de adesão, fato também observado em outras investigações no Brasil ${ }^{31,32}$. Esta situação parece indicar que a maior preocupação dos hipertensos com seu estado de saúde e o acesso e vínculo aos serviços e profissionais de saúde geram maior conhecimento sobre sua condição, o que pode contribuir para um melhor cumprimento da terapia anti-hipertensiva. Desta forma, o acesso aos serviços de saúde e a consultas médicas deve ser garantido, visando minimizar riscos de não adesão ao tratamento.

Cabe ressaltar que a adesão ao tratamento medicamentoso e o incremento das medidas farmacológicas não podem se restringir às consultas médicas. As equipes de saúde da família devem atuar, de forma integrada, na abordagem da avaliação de risco, na adoção de medidas de promoção à saúde e no atendimento aos portadores de hipertensão arterial $^{33}$. As estratégias utilizadas por essas equipes refletem diretamente na demanda dos serviços e nas condições de saúde dos usuários dos serviços e comunidade ${ }^{34}$. Portanto, o planejamento e a execução de suas atividades são fundamentais para minimizar os gastos dos serviços, especialmente os de média e alta complexidade, e, principalmente, para melhorar a qualidade de vida das pessoas ${ }^{14}$.

Quanto às limitações deste estudo, deve-se salientar que a grande maioria das informações obtidas foi autorreferida, o que pode gerar erros decorrentes de memória ou outras distorções. Desta forma, alguns resultados podem estar superestimados, como a própria adesão ao tratamento. Bloch et al. ${ }^{35}$, avaliando a adesão à terapêutica anti-hipertensiva por três diferentes métodos, identificaram que a prevalência de adesão avaliada pelo paciente foi superior à avaliação feita por médicos ou por um questionário validado para a medida da adesão. Assim, os resultados obtidos no presente estudo podem não reproduzir com exatidão os valores de prevalência da adesão à terapia anti-hipertensiva. Os dados apresentados indicam, ainda, uma popula- 
ção com baixa escolaridade e classificação econômica, o que dificulta a extrapolação dos resultados para outros contextos.

Todavia, os resultados encontrados reforçam a importância de ações interdisciplinares que possam contribuir para o manejo e o controle da hipertensão arterial e de outras doenças cardiovasculares. Tais ações devem considerar o indivíduo hipertenso em todas as suas nuances sociais e culturais, valorizando seu ambiente e suas condutas individuais e coletivas ${ }^{36}$. Nesse sentido, estratégias educacionais que desloquem o foco da atenção da produção de procedimentos para a de cuidados integrais são importantes. Uma tecnologia de trabalho em saúde que pode contribuir para uma concepção mais ampliada de saúde é o trabalho em grupo. As oficinas ou grupos de tra-

\section{Colaboradores}

E Girotto, SM de Andrade e MAS Cabrera contribuíram na concepção do projeto, análise e interpretação dos dados e redação do artigo. T Matsuo colaborou na análise e interpretação dos dados, revisão crítica e aprovação da versão final a ser publicada. balho são ferramentas que podem complementar as ações individuais promovidas pelos serviços de saúde, possibilitando que os pacientes compartilhem experiências e vivenciem saberes e práticas que facilitam a escuta, o acolhimento e o vínculo com os profissionais de saúde $e^{37,38}$.

A adoção de uma linha de cuidado no âmbito destes agravos também é importante, com uma rede de serviços que suporte as demandas surgidas e com um projeto terapêutico apropriado a cada usuário, valorizando a integralidade do cuidado, a articulação intersetorial, as condições e necessidades locais e a autonomia dos sujeitos ${ }^{39,40}$. A construção deste processo não deve, então, ser vista apenas pela lógica individual, mas num contexto que envolva toda a sociedade, a família e o próprio usuário no cerne da produção do cuidado.

\section{Referências}

1. Sociedade Brasileira de Cardiologia, Sociedade Brasileira de Hipertensão, Sociedade Brasileira de Nefrologia. VI Diretrizes Brasileiras de Hipertensão Arterial. Arq Bras Cardiol 2010; 95(Supl. 1):1-51.

2. Zaslavsky C, Gus I. Idoso. Doença cardíaca e comorbidades. Arq Bras Cardiol 2002; 79(6):635-639.

3. Alves TCTF, Wajngarten M, Busatto Filho G. Fatores de risco cardiovascular, declínio cognitivo e alterações cerebrais detectadas através de técnicas de neuroimagem. Rev Psiquiatr Clín 2005; 32(3):160-169.

4. Beilin LJ, Puddey IB, Burke V. Lifestyle and hypertension. Am J Hypertens 1999; 12(9):934-945.

5. Chobanian AV, Bakris GL, Black HR, Cushman WC, Green LA, Izzo Junior JL, Jones DW, Materson BJ, Oparil S, Wright Junior JT, Roccella EJ; Joint National Committee on Prevention, Detection, Evaluation, and Treatment of High Blood Pressure. National Heart, Lung, and Blood Institute; National High Blood Pressure Education Program Coordinating Committee. The seventh report of the Joint National Committee on prevention, detection, evaluation, and treatment of high blood pressure. JAMA 2003; 21(19):2560-2572.

6. Leite SN, Vasconcellos MPC. Adesão à terapêutica medicamentosa: elementos para a discussão de conceitos e pressupostos adotados na literatura. Cien Saude Colet 2003; 8(3):775-782.

7. Barbosa RGB, Lima NKC. Índices de adesão ao tratamento anti-hipertensivo no Brasil e mundo. Rev Bras Hipertens 2006; 13(1):35-38.

8. Busnello R, Melchior R, Faccin C, Vettori D, Petter J, Moreira LB, Fuchs FD. Características associadas ao abandono do acompanhamento de pacientes hipertensos atendidos em um ambulatório de referência. Arq Bras Cardiol 2001; 76(5):349-351.

9. Vitor RS, Sakai FK, Consoni PRC. Indicação e adesão de medidas não farmacológicas no tratamento da hipertensão arterial. Rev AMRIGS 2009; 53(2):117-121. 
10. Paiva DCP, Bersusa AAS, Escuder MML. Avaliação da assistência ao paciente com diabetes e/ou hipertensão pelo Programa Saúde da Família do Município de Francisco Morato, São Paulo, Brasil. Cad Saude Publica 2006; 22(2):377-385.

11. Piati J, Felicetti CR, Lopes AC. Perfil nutricional de hipertensos acompanhados pelo Hiperdia em Unidade Básica de Saúde de cidade paranaense. Rev Bras Hipertens 2009; 16(2):123-129.

12. Associação Brasileira de Empresas de Pesquisa (ABEP). CCEB - Critério de classificação econômica Brasil. [acessado 2008 jan 11]. Disponível em: https://www.abep.org/codigosguias/ABEP_CCEB.pdf

13. Baldissera VDA, Carvalho MDB, Pelloso SM. Adesão ao tratamento não-farmacológico entre hipertensos de um centro de saúde escola. Rev Gaúcha Enferm 2009; 30(1):27-32.

14. Brasil. Ministério da Saúde (MS). Política Nacional de Promoção da Saúde. Brasília: MS; 2006. (Série de Pactos pela Saúde, N. 7).

15. Sturmer G, Dias-da-Costa JS, Olinto MTA, Menezes AMB, Gigante DP, Macedo S. O manejo não medicamentoso da hipertensão arterial sistêmica no Sul do Brasil. Cad Saude Publica 2006; 22(8):1727-1737.

16. Contreras EM, Martínez JJC, Won-Vichman MF Guillén VG, Martell N. El incumplimiento terapéutico en el tratamiento de la hipertensión arterial em España. Análisis de los estudios publicados entre 1984 y 2001. Hipertensión 2002; 19(1):12-16.

17. Santa Helena ET, Nemes MIB, Eluf-Neto J. Desenvolvimento e validação de questionário multidimensional para medir não-adesão ao tratamento com medicamentos. Rev Saude Publica 2008; 42(4):764-767.

18. Morisky DE, Green LW, Levine DM. Concurrent and predictive validity of a self-reported measure of medication adherence. Med Care 1986; 24(1):67-74.

19. Spinato IL, Monteiro LZ, Santos ZMSA. Adesão da pessoa hipertensa ao exercício físico - uma proposta educativa em saúde. Texto contexto - enferm. 2010; 19(2):256-264.

20. Pitanga FJG, Lessa I. Prevalência e fatores associados ao sedentarismo no lazer em adultos. Cad Saude Publica 2005; 21(3):870-877.

21. Salles-Costa R, Heilborn ML, Werneck GL, Faerstein E, Lopes CS. Gênero e prática de atividade física de lazer. Cad Saude Publica 2003; 19(Supl. 2): s325-s333.

22. Gomes VB, Siqueira KS, Sichieri R. Atividade física em uma amostra probabilística da população do Município do Rio de Janeiro. Cad Saude Publica 2001; 17(4):969-976.

23. Vinholes DB, Assunção MCF, Neutzling MB. Freqüência de hábitos saudáveis de alimentação me didos a partir dos 10 Passos da Alimentação Saudável do Ministério da Saúde: Pelotas, Rio Grande do Sul, Brasil. Cad Saude Publica 2009; 25(4):791-799.

24. Guedes DP, Gonçalves LAVV. Impacto da prática habitual de atividade física no perfil lipídico de adultos. Arq Bras Endocrinol Metab 2007; 51(1):72-78.

25. Khawali C, Andriolo A, Ferreira SRG. Benefícios da atividade física no perfil lipídico de pacientes com diabetes tipo 1. Arq Bras Endocrinol Metab 2003; 47(1):49-54.

26. Hadi N, Rostami-Gooran N. Determinant factors of medication compliance in hypertensive patients of Shiraz, Iran. Arch Iran Ed. 2004; 7(4):292-296.
27. Vaur L, Vaisse B, Genes N, Elkik F, Legrand C, Poggi L. Use of electronic pill boxes to assess risk of poor treatment compliance: results of a large-scale trial. Am J Hypertens 1999; 12(4):374-380.

28. Yahya AAA, Mehza AMA, Ghareeb HA. Comparison of compliance versus non-compliance to antihypertensive agents in primary health care - an area based study. Kuwait Med J. 2006; 38(1):28-32

29. Berto SJP, Carvalhaes MABL, Moura EC. Tabagismo associado a outros fatores comportamentais de risco de doenças e agravos crônicos não transmissíveis. Cad Saude Publica 2010; 26(8):1573-1582.

30. Costa MFFL, Peixoto SV, César CC, Malta DC, Moura EC. Comportamentos em saúde entre idosos hipertensos, Brasil, 2006. Rev Saude Publica 2009; 43(Supl. 2):18-26.

31. Santa-Helena ET, Nemes MIB, Eluf Neto J. Fatores associados à não-adesão ao tratamento com antihipertensivos em pessoas atendidas em unidades de saúde da família. Cad Saude Publica 2010 26(12):2389-2398

32. Coelho EB, Moysés Neto M, Palhares R, Cardoso MCM, Geleilete TJM, Nobre F. Relação entre a assiduidade às consultas ambulatoriais e o controle da pressão arterial em pacientes hipertensos. Arq Bras Cardiol 2005; 85(3):157-161.

33. Brasil. Ministério da Saúde (MS). Prevenção clínica de doenças cardiovasculares, cerebrovasculares e renais. Brasília: MS; 2006. (Cadernos de Atenção Básica, N. 14).

34. Paula KA, Palha PF, Protti ST. Intersetorialidade: uma vivência prática ou um desafio a ser conquistado? O discurso do sujeito coletivo dos enfermeiros nos núcleos de Saúde da Família do Distrito Oeste Ribeirão Preto. Interface (Botucatu) 2004; 8(15):331348.

35. Bloch KV, Melo AN, Nogueira AR. Prevalência da adesão ao tratamento anti-hipertensivo em hipertensos resistentes e validação de três métodos indiretos de avaliação da adesão. Cad Saude Publica 2008; 24(12):2979-2984.

36. Rangel-S ML. Comunicação no controle de risco à saúde e segurança na sociedade contemporânea: uma abordagem interdisciplinar. Cien Saude Colet 2007; 12(5):1375-1385.

37. Fernandes MTO, Silva LB, Soares SM. Utilização de tecnologias no trabalho com grupos de diabéticos e hipertensos na Saúde da Família. Cien Saude Colet 2011; 16(Supl.1):1331-1341.

38. Silveira LMC, Ribeiro VMB. Grupo de adesão ao tratamento: espaço de "ensinagem" para profissionais de saúde e paciente. Interface (Botucatu) 2005; 9(16):91-104.

39. Malta DC, Merhy EE. O percurso da linha do cuidado sob a perspectiva das doenças crônicas não transmissíveis. Interface (Botucatu) 2010; 14(34):593-605.

40. Silva KL, Sena RR, Seixas CT, Feuerwerker LCM, Merhy EE. Atenção domiciliar como mudança do modelo tecnoassistencial. Rev Saude Publica 2010; 44(1):166-176.

Artigo apresentado em 15/08/2011

Aprovado em 02/11/2011

Versão final apresentada em 28/11/2011 